\title{
Optimal tuning of fractional controllers using genetic algorithms
}

\author{
J.A. Tenreiro Machado
}

Abstract This study addresses the optimization of fractional algorithms for the discrete-time control of linear and non-linear systems. The paper starts by analyzing the fundamentals of fractional control systems and genetic algorithms. In a second phase the paper evaluates the problem in an optimization perspective. The results demonstrate the feasibility of the evolutionary strategy and the adaptability to distinct types of systems.

Keywords Fractional derivatives - Fractional calculus - Optimization - Genetic algorithms

\section{Introduction}

Fractional calculus (FC) deals with the generalization of integrals and derivatives to a non-integer order [14]. In the last decades the application of FC verified a large development in the areas of physics and engineering and we can mention a large volume of research about viscoelasticity, signal processing, diffusion, modeling and control [5-14]. Nevertheless, FC is still considered an 'exotic' mathematical tool and its adoption requires some efforts towards the development of clear algorithms. One of the reasons for this state of affairs is the complexity of the algorithms involved in the calculation of fractional derivatives that require the adoption of approximations for their numerical calculation [15-22]. The area of dynamical systems and control has received a considerable attention from researchers working in FC and recently several papers addressing evolutionary concepts and fractional algorithms can be mentioned [26-30].

Bearing these ideas in mind, this paper addresses the optimal system control using fractional-order algorithms and is organized as follows. Section 2 introduces the calculation of fractional derivatives and formulates the problem of optimization through genetic algorithms (GAs). Section 3 presents a set of experiments that demonstrate the effectiveness of the proposed optimization strategy. Finally, Sect. 4 outlines the main conclusions.

\section{Fundamental concepts and tools}

This section introduces the main mathematical concepts and tools used in the rest of the article. Section 2.1 presents the adopted definition of fractional derivative, the rules for discrete-time calculation and the fractional control algorithm. Section 2.2 outlines the fundamental aspects underlying the GA optimization scheme. 


\subsection{Fractional-order algorithms}

There are several definitions of fractional derivatives. The Riemann-Liouville, the Grünwald-Letnikov, and the Caputo definitions of a fractional derivative of a function $f(t)$ are given by:

$$
\begin{aligned}
{ }_{a} D_{t}^{\alpha} f(t) & =\frac{1}{\Gamma(n-\alpha)} \frac{d^{n}}{d t^{n}} \int_{a}^{t} \frac{f(\tau)}{(t-\tau)^{\alpha-n+1}} d \tau, \\
n-1<\alpha<n & \\
{ }_{a} D_{t}^{\alpha} f(t) & =\lim _{h \rightarrow 0} \frac{1}{h^{\alpha}} \sum_{k=0}^{\left[\frac{t-a}{h}\right]}(-1)^{k}\left(\begin{array}{l}
\alpha \\
k
\end{array}\right) f(t-k h) \\
{ }_{a} D_{t}^{\alpha} f(t) & =\frac{1}{\Gamma(\alpha-n)} \int_{a}^{t} \frac{f^{(n)}(\tau)}{(t-\tau)^{\alpha-n+1}} d \tau, \\
n-1<\alpha<n &
\end{aligned}
$$

where $\Gamma()$ is Euler's gamma function, $[x]$ means the integer part of $x$, and $h$ is the step time increment.

It is also possible to generalize several results based on transforms, yielding expressions such as the Laplace expression:

$$
L\left\{{ }_{0} D_{t}^{\alpha} f(t)\right\}=s^{\alpha} L\{f(t)\}-\sum_{k=0}^{n-1} s_{0}{ }_{0} D_{t}^{\alpha-k-1} f 0^{+}
$$

where $s$ and $L$ represent the Laplace variable and operator, respectively.

For a wide class of functions which appear in real physical and engineering applications, these definitions are equivalent. The expressions lead to the standard results when $\alpha=1$ and demonstrate that fractional derivatives have memory, contrary to integer derivatives that consist in local operators.

There is a long-standing discussion, still going on, about the pros and cons of the different definitions. These debates are outside the scope of this paper, but, in short, while the Riemann-Liouville definition involves an initialization of fractional order, the Caputo counterpart requires integer order initial conditions which are easier to apply (often the Caputo initial conditions are called freely as 'with physical meaning'). The Grünwald-Letnikov formulation is frequently adopted in numerical algorithms and control systems because it inspires a discrete-time calculation algorithm, based on the approximation of the time increment $h$ through the sampling period $T$, yielding the equation in the $z$ domain:

$\left.Z\left\{D^{\alpha} f(t)\right\} \approx \frac{1}{T^{\alpha}} \sum_{k=0}^{\infty} \frac{(-1)^{k} \Gamma(\alpha+1)}{k ! \Gamma(\alpha-k+1)} z^{-k}\right] F(z)$

where $F(z)=Z\{f(t)\}$.

The implementation of expression (5) corresponds to an $r$-term truncated series given by:

$$
\left.Z\left\{D^{\alpha} f(t)\right\} \approx \frac{1}{T^{\alpha}} \sum_{k=0}^{r} \frac{(-1)^{k} \Gamma(\alpha+1)}{k ! \Gamma(\alpha-k+1)} z^{-k}\right] F(z)
$$

Expression (5) represents the Euler (or first backward difference) approximation in the so-called $s \rightarrow z$ conversion scheme. Another possibility, often adopted in control system design, consists in the Tustin (or bilinear) rule. The Euler and Tustin rational expressions, $H_{0}\left(z^{-1}\right)=\frac{1}{T}\left(1-z^{-1}\right)$ and $H_{1}\left(z^{-1}\right)=\frac{2}{T} \frac{1-z^{-1}}{1+z^{-1}}$, are often called generating approximants of zero and first order, respectively. Therefore, the generalization of these conversion methods leads to the non-integer or$\operatorname{der} \alpha$ results:

$s^{\alpha} \approx \frac{1}{T} 1-z^{-1}=H_{0}^{\alpha} z^{-1}$

$s^{\alpha} \approx\left(\frac{2}{T} \frac{1-z^{-1}}{1+z^{-1}}\right)^{\alpha}=H_{1}^{\alpha} z^{-1}$

We can obtain a family of fractional differentiators generated by $H_{0}^{\alpha}\left(z^{-1}\right)$ and $H_{1}^{\alpha}\left(z^{-1}\right)$ weighted by the factors $p$ and $1-p$, yielding:

$H_{a v}\left[z^{-1} ;(p, \alpha)\right]=p H_{0}^{\alpha}\left(z^{-1}\right)+(1-p) H_{1}^{\alpha}\left(z^{-1}\right)$

In order to get a rational expression, the final approximation corresponds to a truncated Taylor series or a rational fraction expansion. Due to its superior performance often it is used a fraction:

$H_{k} z^{-1}=\frac{\sum_{i=0}^{k} a_{i} z^{-i}}{\sum_{i=0}^{k} b_{i} z^{-i}}, \quad a_{i}, b_{i} \in \Re$

where $k \in \aleph$ denotes the order of the approximation. Moreover, usually is adopted a Padé expansion in the neighborhood of $z^{-1}=0$ and, since one parameter is linearly dependent, it is established that $b_{0}=1$. 
The FC concepts can be adopted in control theory, and a typical case is the generalization of the classical PID controller. The fractional PID (FrPID), or $P I^{\lambda} D^{\mu}$, consists in a control algorithm with the integer integral and derivative of the $I$ and $D$ actions replaced by their fractional generalizations, yielding the transfer function:

$G_{c}(s)=K_{p}+K_{i} s^{-\lambda}+K_{d} s^{\mu}, \quad 0<\lambda, \mu \leq 1$

where $s$ represents the Laplace variable, $0<\lambda, \mu \leq$ 1 are the fractional orders, and $\left\{K_{p}, K_{i}, K_{d}\right\}$ denote the proportional, integral and derivative gains, respectively.

\subsection{Optimization through genetic algorithms}

A GA is a computational technique to find exact or approximate solutions of optimization problems [23-25]. GAs are simulated in a computing system, and consist in a population of representations of candidate solutions, of an optimization problem, that evolve toward better solutions.

Once the genetic representation and the fitness function are defined, the GA proceeds to initialize a population of solutions randomly, and then to improve it through the repetitive application of mutation, crossover and selection operators.

The evolution usually starts from a population of randomly generated individuals. In each generation, not only the fitness of every individual in the population is evaluated, but also several individuals are stochastically selected from the current population and modified to form a new population. The new population is then used in the next iteration of the algorithm. The GA terminates when either the maximum number of generations $N$ is produced, or a satisfactory fitness level has been reached.

During the successive generation, a part or the totality of the population is selected to breed a new generation. Individual solutions are selected through a fitness-based process, where fitter solutions (measured by a fitness function) are usually more likely to be selected. The pseudo-code of the GA is:

1. Choose the initial population

2. Evaluate the fitness of each individual in the population

3. Repeat

3.1 Select best-ranking individuals to reproduce

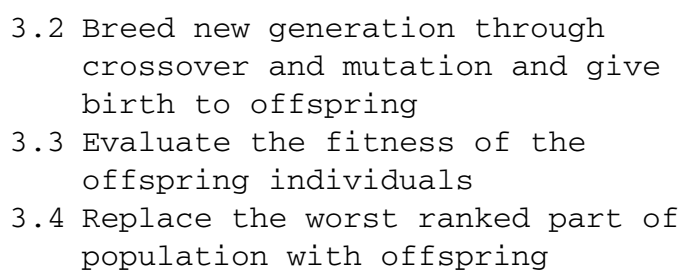

The present article adopts also the common technique of elitism, which is the process of selecting the better individuals to form the parents in the offspring generation.

\section{Fractional-order differentiation}

In this section we study system optimal control using fractional algorithms tuned through GAs.

We start by defining an appropriate optimization index in the perspective of system control. We consider the integral squared error (ISE) defined as:

$I S E=\int_{0}^{T} e^{2}(t) d t$

where $t$ denotes the time variable, $e(t)$ represents the closed-loop control system error and $T$ is a time period sufficiently long for settling the response close to steady-state. Other optimization indexes, such as the IAE, ITAE and ITSE, were tested leading to the same type of results and, therefore, in the sequel the analysis will concentrate merely on the ISE index.

In the second place we define a simple prototype dynamical system for supporting the simulations. We adopt the open-loop plant with transfer function:

$G_{p}(s)=\frac{K}{s(s+\tau)}$

where $K$ and $\tau$ are the open-loop system gain and time constant, respectively.

Since this linear plant constitutes a simple challenge to the controller we consider also a second system for comparison, namely the plant (12) including static backlash nonlinearity of width $h[31,32]$.

In the closed-loop system is adopted a fractionalorder control algorithm. The standard FrPID adopts three terms representative of the proportional, integral and derivative actions. However, in general, a different number of terms, either larger or smaller, can be the most adequate for the optimal control of a system with 
a given set of dynamical characteristics. Therefore, we adopt a more general algorithm given by:

$G_{c}(s)=\sum_{i=1}^{n} K_{i} s^{\alpha_{i}}$

where $n \geq 1$ is the number of control actions of fractional order $-1 \leq \alpha_{i} \leq 1$ and gain $K_{i} \geq 0$.

The closed-loop system consists in plant (12), with or without backlash, under the action of algorithm (11), where the number of components $n$ is not fixed a priori, but has to be calculated when optimizing the ISE index (11). Furthermore, it is considered a unit feedback and the standard case of optimization for a unit step reference input for the closed-loop system.

Finally we must define the characteristics of the GA scheme for performing the optimization in the viewpoint of fitness (11). The controller fractional actions are implemented through (8)-(9) for a weight $p=$ $3 / 4$. Each gene in the chromosome represents the gain and order pair $\left(K_{i}, \alpha_{i}\right), i=1, \ldots, n$. In order to avoid a combinatorial explosion of terms in (13), in the GA was adopted a discretization of the fractional domain by defining 200 classes in the interval $-1 \leq \alpha_{i} \leq 1$. Therefore, two fractional orders less than $2.5 \%$ apart are rounded to the same value. Furthermore, two control components in (13) with identical orders are converted to a single one, that is, pairs such as $\left(K_{1 a}, \alpha_{1}\right)$ and $\left(K_{1 b}, \alpha_{1}\right)$ are simplified to $\left(K_{1 a}+K_{1 b}, \alpha_{1}\right)$ while the remaining control action is reset to $(0,0)$.

A given set of values in a GA-solution can lead to an unstable closed-loop response. Therefore, the state variables are checked during the control system time simulation (there was adopted the Runge-Kutta 4 and a maximum of $T=10$ seconds) and once a threshold limit is reached, the system is considered unstable. In this case, the simulation of that case is abandoned and a new set of values is generated randomly substituting the previous element of the GA population.

The experiments demonstrated some difficulties in the GA acquiring the optimal solution. Consequently, several measures were adopted to overcome that problem, namely, a large GA population with $P=500$ elements, the crossover of all population elements and the adoption of elitism, a mutation probability of $8 \%$, and an evolution with $N=100$ iterations. Even so, it was observed that after some iteration the GA tended to stabilize in sub-optimal solutions and other values for the GA parameters had no significant impact. Therefore, a complementary strategy was taken to prevent

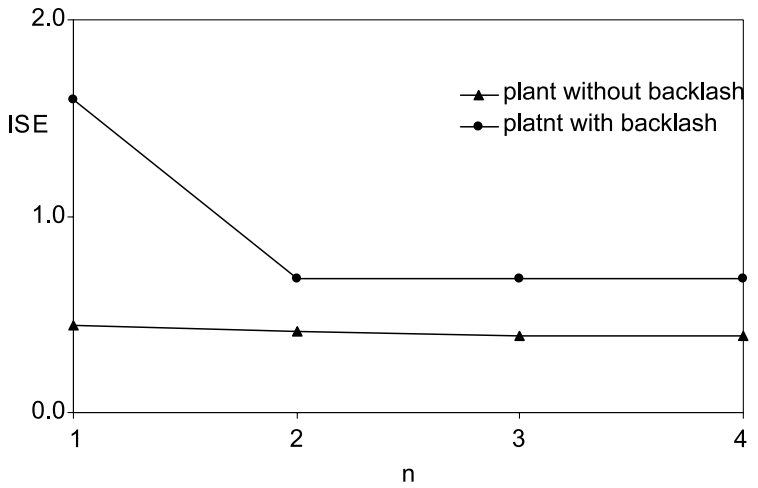

Fig. 1 Evolution of the ISE versus $n$ for the closed-loop system consisting in plant (12), $K=1, \tau=1$, without and with backlash, and the control algorithm (13)

such behavior, by implementing a hierarchical GA with two loops. The inner loop, in a base level, consists in the GA described previously, while the outer loop, at a higher level, restarts the base GA population including the best solutions obtained so far. In other words, the base GA is repeated by including in its population the best solutions from previous executions and the hierarchical process stops only when two GA executions stabilize in identical final solutions.

Figure 1 depicts the evolution of the ISE index versus $n$ for the closed-loop system with (12)-(13) ( $K=$ $1, \tau=1)$ both without and with backlash $(h=0.5)$. Figures 2 and 3 depict the step response for the closedloop system under the action of the fractional algorithm without and with backlash for $n=1,2$. We verify that, as expected, the system with the nonlinearity reveals a larger value of ISE. We observe also that $n=1$ is the worst case and that the controller converges rapidly to the adequate number of terms in (13). It should be noted that the numbers of terms in the two experiments is small, but it is not possible to generalize and other systems and nonlinearities may require more terms. In all cases it is straightforward to apply the hierarchical GA that yields the best number of terms and the optimal tuning.

\section{Conclusions}

The recent advances in fractional calculus point towards important developments in the application of this mathematical concept. During the last years several algorithms for the application of fractional derivatives in control were proposed. The resulting systems 
Fig. 2 Closed-loop time response for $n=1,2$ and plant (12), $K=1, \tau=1$, without backlash
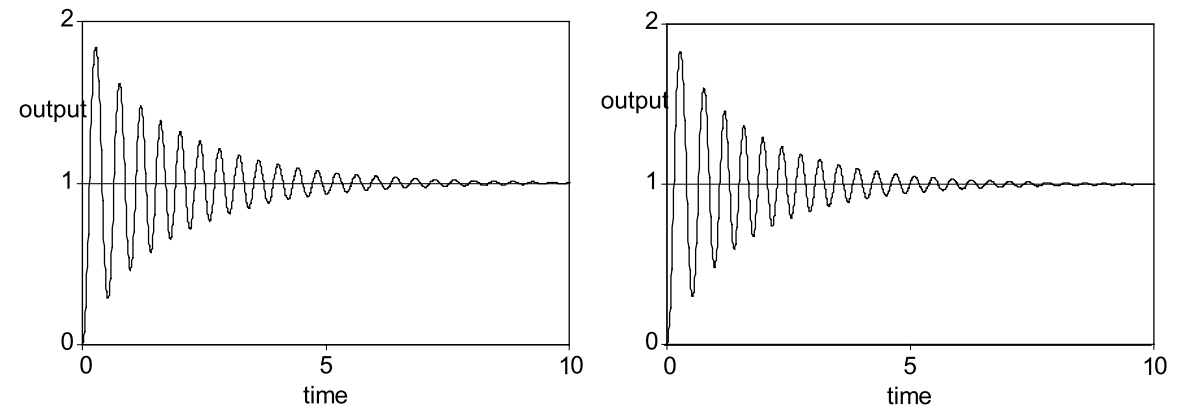

Fig. 3 Closed-loop time response for $n=1,2$ and plant (12), $K=1, \tau=1$, with backlash $h=0.5$
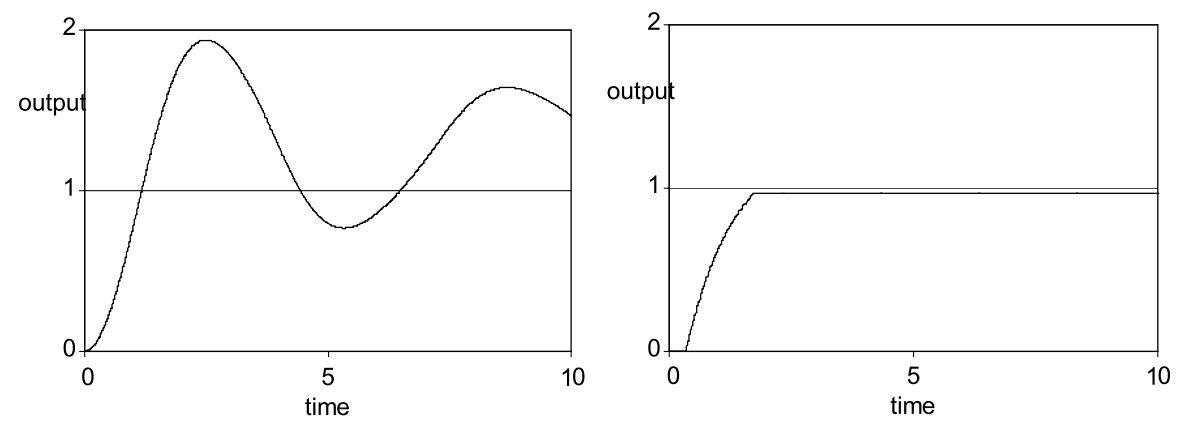

are non-optimal revealing the controller design should be formulated as an optimization problem. In this paper a new method, based on evolutionary concepts, for the calculation of fractional control algorithms, was studied. The results demonstrate the excellent performance and the adaptability to different types of systems.

Acknowledgements The author would like to thank the anonymous reviewers for their comments, which helped to improve the paper.

\section{References}

1. Oldham, K.B., Spanier, J.: The Fractional Calculus: Theory and Application of Differentiation and Integration to Arbitrary Order. Academic Press, San Diego (1974)

2. Samko, S.G., Kilbas, A.A., Marichev, O.I.: Fractional Integrals and Derivatives: Theory and Applications. Gordon and Breach, New York (1993)

3. Miller, K.S., Ross, B.: An Introduction to the Fractional Calculus and Fractional Differential Equations. Wiley, New York (1993)

4. Podlubny, I.: Fractional Differential Equations. Academic Press, San Diego (1999)

5. Bagley, R.L., Torvik, P.J.: Fractional calculus-a different approach to the analysis of viscoelastically damped structures. AIAA J. 21, 741-748 (1983)

6. Oustaloup, A.: La Commande CRONE: Commande Robuste d'Ordre non Entier. Hermes, Paris (1991)
7. Anastasio, T.J.: The fractional-order dynamics of brainstem vestibulo-oculomotor neurons. Biol. Cybern. 72(1), 69-79 (1994)

8. Mainardi, F.: Fractional relaxation-oscillation and fractional diffusion-wave phenomena. Chaos Solitons Fractals 7, 1461-1477 (1996)

9. Tenreiro Machado, J.A.: Analysis and design of fractionalorder digital control systems. J. Syst. Anal. Model. Simul. 27, 107-122 (1997)

10. Nigmatullin, R.: The statistics of the fractional moments: Is there any chance to "read quantitatively" any randomness? Signal Process. 86(10), 2529-2547 (2006)

11. Tarasov, V.E., Zaslavsky, G.M.: Fractional dynamics of systems with long-range interaction. Commun. Nonlinear Sci. Numer. Simul. 11(8), 885-898 (2006)

12. Sabatier, J., Agrawal, O.P., Tenreiro Machado, J.A. (eds.): Advances in Fractional Calculus. Theoretical Developments and Applications in Physics and Engineering. Springer, Berlin (2007). ISBN:978-1-4020-6041-0

13. Tenreiro Machado, J.A.: Fractional derivatives: probability interpretation and frequency response of rational approximations. Commun. Nonlinear Sci. Numer. Simul. 14(910), 3492-3497 (2009)

14. Baleanu, D.: About fractional quantization and fractional variational principles. Commun. Nonlinear Sci. Numer. Simul. 14(6), 2520-2523 (2009)

15. Podlubny, I.: Fractional-order systems and $\mathrm{PI}^{\lambda} \mathrm{D}^{\mu}-$ controllers. IEEE Trans. Autom. Control 44(1), 208-213 (1999)

16. Tenreiro Machado, J.A.: Discrete-time fractional-order controllers. J. Fract. Calc. Appl. Anal. 4, 47-66 (2001)

17. Chen, Y.Q., Moore, K.L.: Discretization schemes for fractional-order differentiators and integrators. IEEE Trans. 
Circuits Syst. I, Fundam. Theory Appl. 49(3), 363-367 (2002)

18. Tseng, C.C.: Design of fractional order digital fir differentiators. IEEE Signal Process. Lett. 8(3), 77-79 (2001)

19. Vinagre, B.M., Chen, Y.Q., Petras, I.: Two direct Tustin discretization methods for fractional-order differentiator/integrator. J. Franklin Inst. 340(5), 349-362 (2003)

20. Chen, Y.Q., Vinagre, B.M.: A new IIR-type digital fractional order differentiator. Signal Process. 83(11), 23592365 (2003)

21. Barbosa, R.S., Tenreiro Machado, J.A., Silva, M.: Time domain design of fractional differintegrators using least squares approximations. Signal Process. 86(10), 25672581 (2006)

22. Al-Alaoui, M.A.: Novel digital integrator and differentiator. Electron. Lett. 29(4), 376-378 (1993)

23. Holland, J.H.: Adaptation in Natural and Artificial Systems. University of Michigan Press, Ann Arbor (1975)

24. Goldenberg, D.E.: Genetic Algorithms in Search Optimization, and Machine Learning. Addison-Wesley, Reading (1989)

25. Tenreiro Machado, J.A.: Calculation of fractional derivatives of noisy data with genetic algorithms. Nonlinear Dyn. 57(1-2), 253-260 (2009)

26. Tenreiro Machado, J.A., Galhano, A., Oliveira, A.M., Tar, J.K.: Optimal approximation of fractional deriva- tives through discrete-time fractions using genetic algorithms. Commun. Nonlinear Sci. Numer. Simul. 15, 482490 (2010)

27. Maiti, D., Acharya, A., Chakraborty, M., Konar, A., Janarthanan, R.: Tuning PID and $\mathrm{PI}^{\lambda} \mathrm{D}^{\delta}$ controllers using the integral time absolute error criterion. In: IEEE Forth International Conference on Information and Automation for Sustainability, December 12-14, 2008, Colombo, Sri Lanka

28. Cao, J.-Y., Cao, B.-G.: Design of fractional order controller based on particle swarm optimization. Int. J. Control Autom. Syst. 4(6), 775-781 (2006)

29. Valério, D., Sá da Costa, J.: Tuning of fractional controllers minimising $\mathrm{H}_{2}$ and $\mathrm{H}_{\infty}$ norms. Acta Polytech. Hung. 3(4), 55-70 (2006)

30. Biswas, A., Das, S., Abraham, A., Dasgupta, S.: Design of fractional-order $\mathrm{PI}^{\lambda} \mathrm{D}^{\mu}$ controllers with an improved differential evolution. Eng. Appl. Artif. Intell. 22, 343-350 (2009)

31. Barbosa, R.S., Tenreiro Machado, J.A.: Describing function analysis of systems with impacts and backlash. Nonlinear Dyn. 29(1-4), 235-250 (2002)

32. Duarte, F., Tenreiro Machado, J.A.: Describing function of two masses with backlash. Nonlinear Dyn. 56(4), 409-413 (2009) 